\title{
Deconfinement and Chiral Restoration in Hot and Dense Matter
}

\author{
Kenji Fukushima * a b \\ ${ }^{a}$ Center for Theoretical Physics, MIT, 77 Mass. Ave., Cambridge, MA 02139, USA \\ ${ }^{\mathrm{b}}$ Department of Physics, University of Tokyo, 7-3-1 Hongo, Bunkyo-ku, Tokyo 113-0033, Japan
}

\begin{abstract}
We propose a picture that the chiral phase transition at zero quark mass and the deconfinement transition at infinite quark mass are continuously connected. This gives a simple interpretation on the coincidence of the pseudo-critical temperatures observed in lattice QCD. We discuss a possible dynamical mechanism behind the simultaneous crossovers and show the results in a model study.
\end{abstract}

It is widely accepted that Quantum Chromodynamics (QCD) has phase transitions at high temperature, the nature of which depends on the quark mass, $m_{\mathrm{q}}$. In the limit of $m_{\mathrm{q}}=0, \mathrm{QCD}$ has chiral symmetry, $\mathrm{SU}_{\mathrm{L}}\left(N_{\mathrm{f}}\right) \times \mathrm{SU}_{\mathrm{R}}\left(N_{\mathrm{f}}\right)$, in the case of $N_{\mathrm{f}}$ flavors, that spontaneously breaks into $\mathrm{SU}_{\mathrm{V}}\left(N_{\mathrm{f}}\right)$ at low temperatures. The chiral condensate, $\langle\bar{q} q\rangle$, serves as an order parameter for chiral restoration at high temperature. Since the quark mass term breaks chiral symmetry, $m_{\mathrm{q}} / f_{\pi}$ is regarded as the magnitude of the explicit chiral symmetry breaking. When $m_{\mathrm{q}}=\infty$, on the other hand, there is no dynamical quark and thus no remnant of chiral symmetry at all. QCD is reduced to a pure gluonic theory, which has center symmetry at finite temperature, that is $\mathrm{Z}\left(N_{\mathrm{c}}\right)$ for $N_{\mathrm{c}}$ colors. The order parameter is given by the Polyakov loop, $\langle l\rangle=\langle\operatorname{tr} L\rangle=\left\langle\operatorname{tr} \mathcal{T} \mathrm{e}^{-\mathrm{i} \int \mathrm{d} x_{4} A_{4}}\right\rangle$. Center symmetry is broken by thermal quark excitation and the magnitude of the explicit center symmetry breaking is $\mathrm{e}^{-M_{\mathrm{q}} / T}$ where $M_{\mathrm{q}}$ is the constituent quark mass.

In the limits of $m_{\mathrm{q}}=0$ and $m_{\mathrm{q}}=\infty$ the nature of the QCD phase transition can be predicted from generic arguments based on the universality class. It has been almost established today that the chiral phase transition at $m_{\mathrm{q}}=0$ is of second order for $N_{\mathrm{f}}=2$ and first order for $N_{\mathrm{f}} \geq 3$ [1], and that the deconfinement phase transition at

\footnotetext{
*We acknoledge the support of the Japan Society for the Promotion of Science for Young Scientists and the U.S. Department of Energy under cooperative research agreement \#DF-FC02-94ER40818.
}

$m_{\mathrm{q}}=\infty$ is of second order for $N_{\mathrm{c}}=2$ and first order for $N_{\mathrm{c}} \geq 3[2]$. Thus, a question arises; what is the QCD phase transition for $0<m_{\mathrm{q}}<\infty$ ?

$A$ naive expectation would be the following. The deconfinement and chiral phase transitions are different phenomena lying in the opposite limits. The critical temperature is known from lattice QCD to be $T_{\chi} \simeq 150 \mathrm{MeV}$ for chiral restoration and $T_{\mathrm{d}} \simeq 270 \mathrm{MeV}$ for deconfinement. In the presence of finite $m_{\mathrm{q}}$ they are both blurred by the explicit symmetry breaking. One would, as a result, expect to find a crossover associated with chiral restoration near $T_{\chi}$ and another crossover associated with deconfinement near $T_{\mathrm{d}}$.

The fact turned out different. In the lattice QCD simulation the chiral condensate and the Polyakov loop have been measured as a function of the temperature. Contrary to the naive expectation, only one crossover has been found. The chiral condensate and the Polyakov loop indicate a crossover simultaneously at the same temperature, $T_{\mathrm{c}}$, and moreover, the chiral susceptibility has one peak at $T=T_{\mathrm{c}}$, and so does the Polyakov loop susceptibility [3].

A naive explanation would be the following. Since there is only one crossover, there must be either the chiral or deconfinement phase transition in reality. A peak in the susceptibility signifies a remnant of the second-order phase transition in which the susceptibility has a singularity at the critical point. One might think that only the chiral phase transition can be regarded as an approximate second-order phase transition, so that 


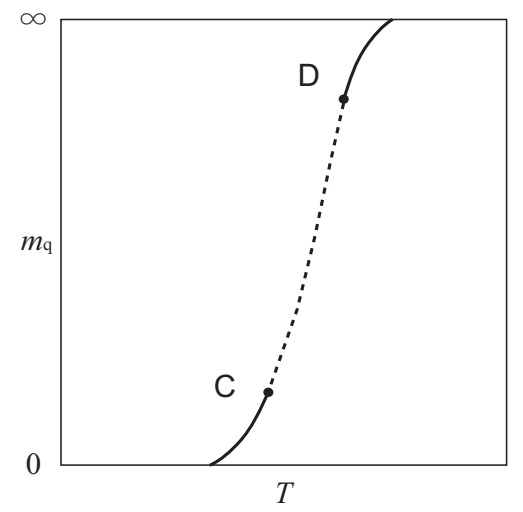

Figure 1. Schematic picture of the QCD phase transition taken from Ref. 7].

the susceptibility peak comes only from the chiral phase transition and no remnant of the deconfinement transition remains. This explanation is not correct, however.

Even though the above explanation could work in the vicinity of the chiral limit, the lattice data is taken for various quark masses corresponding to the pion mass ranging from $\sim 400 \mathrm{MeV}$ to several $\mathrm{GeV}$, that are not so close to the chiral limit. It could be even possible that the lattice data is closer to the deconfinement transition rather than the chiral phase transition. The point is that there is a second-order phase transition not only near the chiral limit but also near the heavy quark mass limit. In the case of $N_{\mathrm{f}}=3$ and $N_{\mathrm{c}}=3$, as mentioned before, both the chiral and deconfinement phase transitions in the limit of $m_{\mathrm{q}}=0$ and $m_{\mathrm{q}}=\infty$ are of first order. The phase transition is smeared by the effect of the explicit symmetry breaking and eventually becomes a crossover. The point where the phase transition ceases to be of first order is called the critical end-point (CEP), at which the system undergoes a secondorder phase transition. Interestingly enough, the lattice and model studies give the quark mass around $800 \mathrm{MeV}$ at the deconfinement CEP, that seems not to be very heavy 456 .

$A$ correct interpretation on the lattice data should be the following. One must consider both the chiral and deconfinement transitions on an equal footing. When $m_{\mathrm{q}}$ is small, there is a second-order phase transition at the chiral CEP, denoted by $\mathrm{C}$, and a second-order phase transition at the deconfinement CEP, denoted by $\mathrm{D}$ in Fig. 11 The susceptibility peak may reflect $C$ or $D$ or possibly both for a quark mass of order hundreds $\mathrm{MeV}$. The fact is, however, there appears only one peak in the susceptibility for all $m_{\mathrm{q}}$. This means that C and D are connected smoothly by a single crossover boundary as shown by the dashed curve in Fig. 1 17. We would emphasize that this is the only one possible interpretation based on the fact observed on the lattice.

The nature of the QCD phase transition is highly non-trivial rather than what has been argued naively. There is one phase transition that is a mixture of chiral restoration and deconfinement, neither of which loses its physical meaning. Then, a question one will come across next would be how two distinct phenomena become locked together dynamically. Since no generic argument is applicable due to the explicit breaking of chiral and center symmetries, it should be a dynamical problem depending on the coupling between the chiral condensate (or the $\sigma$ meson) and the Polyakov loop.

The coupling proposed in Ref. [6] is given by

$$
\begin{aligned}
\sim \int \frac{\mathrm{d}^{3} p}{(2 \pi)^{3}} \operatorname{Tr}_{\mathrm{c}}\left\{\ln \left[1+L \mathrm{e}^{-\left(E_{p}-\mu\right) / T}\right]\right. \\
\left.+\ln \left[1+L^{\dagger} \mathrm{e}^{-\left(E_{p}+\mu\right) / T}\right]\right\},
\end{aligned}
$$

where $\operatorname{Tr}_{\mathrm{c}}$ is the trace over color and $\mu$ a quark chemical potential. The quasi-quark energy is $E_{p}=\sqrt{p^{2}+M_{\mathrm{q}}^{2}}$ where $M_{\mathrm{q}}^{2}$ depends on the chiral condensate, which gives the coupling between the chiral condensate and the Polyakov loop.

Fig. 2 shows the results from a model for the chiral condensate and the Polyakov loop with the coupling (11). The deconfinement transition is, as shown by the dotted curve, of first order without dynamical quarks. The chiral condensate shown by another dotted curve is the result of the standard NJL model with $u$ and $d$ quarks having the mass, $m_{\mathrm{q}}=5.5 \mathrm{MeV}$. They are shown for reference. Obviously the chiral crossover (solid curve) 


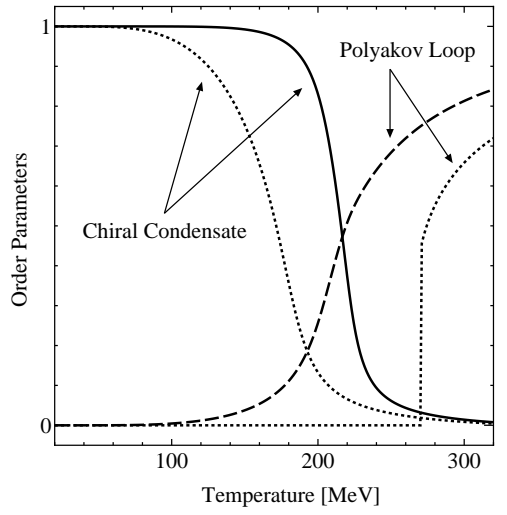

Figure 2. The chiral condensate and the Polyakov loop as a function of the temperature.

and the deconfinement crossover (dashed curve) occur around the same pseudo-critical temperature as a result of the coupling (11).

The reason two crossovers come closer to each other is understood from the generic property of the coupling (11). Roughly speaking, if the expectation value of the Polyakov loop is small, the quark excitation is suppressed because $L$ is in front of the thermal factor of the quark excitation. This means that chiral restoration cannot occur as long as the Polyakov loop stays small. Once the Polyakov loop grows with increasing temperature, the chiral condensate decreases, leading to the simultaneous crossovers. This handwaving argument can be sophisticated in a more wellfounded model. In the Gocksch-Ogilvie model, which is derived from the lattice QCD in the strong coupling and large dimensional expansion, it has been shown that the chiral condensate is always finite if the Polyakov loop is forced to be zero by hand 5 .

Fig. 3 is a prediction for the finite density case from our model. The density is chosen at the chiral CEP, namely $\mu=321 \mathrm{MeV}$ in this model. The temperature slope of the chiral condensate diverges at $T=106 \mathrm{MeV}$. The Polyakov loop has a longer tail because denser quark matter has more quarks breaking the center symmetry. In this case it is hard to say that two crossovers are

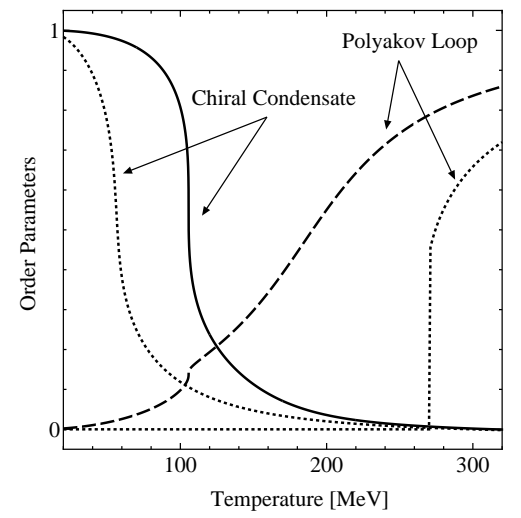

Figure 3. The chiral condensate and the Polyakov loop at $\mu=321 \mathrm{MeV}$.

simultaneous. In the future lattice simulation at finite density, this prediction would be tested.

Although this model goes well for small $m_{\mathrm{q}}$, the level repulsion discussed in Ref. [7] is not strong enough to lead to the perfect locking between the chiral and deconfinement phase transitions for all $m_{\mathrm{q}}$, as shown in Fig. 1] It is still an open question to clarify the full dynamics linking the chiral and deconfinement phase transitions entirely.

The work involving Fig. 1 was performed in collaboration with Yoshitaka Hatta.

\section{REFERENCES}

1. R.D. Pisarski and F. Wilczek, Phys. Rev. D 29 (1984) 338.

2. B. Svetitsky and L.G. Yaffe, Nucl. Phys. B 210 [FS6] (1982) 423.

3. M. Fukugita and A. Ukawa, Phys. Rev. Lett. 57 (1986) 503; F. Karsch and E. Laermann, Phys. Rev. D 50 (1994) 6954; S. Aoki et al., Phys. Rev. D 57 (1998) 3910.

4. N. Attig, B. Petersson, M. Wolff and R.V. Gavai, Z. Phys. C 40 (1988) 471.

5. K. Fukushima, Phys. Lett. B 553 (2003) 38, Phys. Rev. D 68 (2003) 045004.

6. K. Fukushima, Phys. Lett. B 591 (2004) 277.

7. Y. Hatta and K. Fukushima, Phys. Rev. D 69 (2004) 097502, hep-ph/0311267 